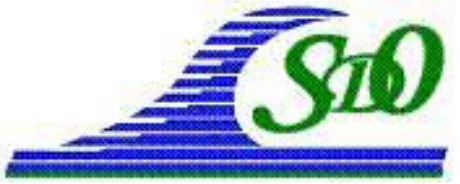

\title{
Dynamique de la crème de vase dans l'estuaire de la Loire
}

\section{Franck DESMAZES ${ }^{1}$, Aldo SOTTOLICHIO ${ }^{2}$, Christine BERTIER ${ }^{3}$, Jean-Baptiste LIBAUD ${ }^{1}$, Malory BECHE ${ }^{1}$}

1. HOCER, 1 place de Strasbourg, 29200 Brest, France.

franck.desmazes@hocer.com

2. Université Bordeaux 1, UMR CNRS EPOC 5805, avenue des Facultés, 33405 Talence cedex, France.

a.sottolichio@epoc.u-bordeauxl.fr

3. GIP Loire Estuaire, 22 rue de la Tour d'Auvergne, 44200 Nantes, France.

christine.bertier@loire-estuaire.org

\section{Résumé :}

Cette étude a pour but de mettre à jour la caractérisation des dépôts de crème de vase dans l'estuaire de Loire, à partir des bandes imprimées lors de sondages effectués régulièrement par le Grand Port Maritime de Nantes-Saint Nazaire entre 1998 et 2008. Les bandes de sondage, matière première de ce travail, correspondent à des mesures bathymétriques issues d'un sondeur bifréquence et acquises au cours de transits (le long de l'estuaire) et de profils transversaux dans le chenal de navigation. Dans un premier temps, l'ensemble des bandes de sondage a été regardé pour déterminer la présence ou non de crème de vase. Dans un second temps, la position, la longueur et les caractéristiques du signal acoustique des lentilles ont été étudiées pour les situations présentant de la crème de vase. Dans un troisième temps, une large variété de situations a été analysée finement afin de détailler l'épaisseur de chaque faciès acoustique observé. En décrivant un éventail varié de situations, cette étude a permis d'améliorer la connaissance des dépôts de crème de vase et de leur dynamique dans l'estuaire de la Loire. Des correspondances entre les caractéristiques de dépôts, les conditions hydrologiques et la géométrie du chenal ont pu être établies.

Mots-clés :

Sédiments fins - Crème de vase - Milieu estuarien - Sondeur bifréquence Hydrodynamique sédimentaire

\section{Introduction}

La crème de vase est un dépôt de vase fluide, qui constitue un état distinct et intermédiaire entre l'eau turbide et la vase consolidée (KIRBY, 1988). Ces sédiments fluides, fréquemment rencontrés dans les estuaires, sont particulièrement mobiles, souvent associés à la présence du bouchon vaseux (ROSS \& MEHTA, 1989). Dans l'estuaire de la Loire, la formation de la crème de vase et sa remobilisation peuvent avoir des conséquences sur l'oxygénation des eaux (MAURICE, 1994). Sa persistance 
dans les chenaux et les ports ainsi que sa consolidation progressive constituent une gêne pour la navigation des plus gros navires (SANCHEZ \& DELANOË, 2006), ce qui nécessite la mise en place de lourds travaux de dragage. Pour établir des scénarios d'aménagement durable de l'estuaire, qui permettent d'évoluer vers une dynamique sédimentaire moins pénalisante, il est nécessaire de mieux connaître les caractéristiques des dépôts de vase et de crème de vase dans la Loire.

L'estuaire de la Loire s'étend sur $110 \mathrm{~km}$ depuis Ancenis jusqu'à son embouchure dans l'océan Atlantique au large de Saint-Nazaire. D'un point de vue morpho-sédimentaire, l'estuaire se divise en trois zones. A l'ouest du rétrécissement de Saint-Nazaire, l'estuaire externe ou zone d'étude aval est un vaste golfe dont les fonds sont de nature sableuse ou sablo-vaseuse. Au milieu, la partie centrale de l'estuaire est caractérisé par un fond essentiellement vaseux, mais aussi par une diversité d'environnements : zone d'îles et de bancs sableux entre Saint Nazaire et Paimboeuf, domaine de marais, entre Paimboeuf et le PK50, zone endigué entre le PK50 et Nantes. A l'est du seuil de Bellevue (amont immédiat de Nantes), dans l'estuaire interne, la pente du lit est beaucoup plus raide. Dans ce bief, la Loire est constituée d'un réseau hydraulique plus complexe formé d'îles et de bras secondaires. L'étude a été menée sur les zones de présence préférentielle de la crème de vase, dans les parties externe et centrale (Fig. 1). D'un point de vue hydrodynamique, l'estuaire de la Loire est soumis à une marée méso à macrotidale, dont le marnage en vives-eaux extrêmes est supérieur à $6 \mathrm{~m}$, et à un débit fluvial moyen de $850 \mathrm{~m}^{3} / \mathrm{s}$ (MIGNIOT, 1993). L'importance des dépôts fins dans les vasières, les ports, les darses et le chenal de navigation est liée à la présence d'un bouchon vaseux, zone de très forte turbidité. La position du bouchon vaseux évolue dans l'ensemble de l'estuaire en fonction du débit fluvial et des coefficients de marée.

La caractérisation de la dynamique longitudinale et saisonnière de la crème de vase en Loire date du milieu des années 1970 (GALLENNE, 1974). La présente étude, réalisée par la société HOCER et l'Université Bordeaux 1 pour le GIP Loire Estuaire, a pour but d'actualiser cette connaissance sur la base des observations les plus récentes réalisées par le Port de Nantes et de la compléter en étudiant les caractéristiques de concentration, position et extension de la crème de vase.

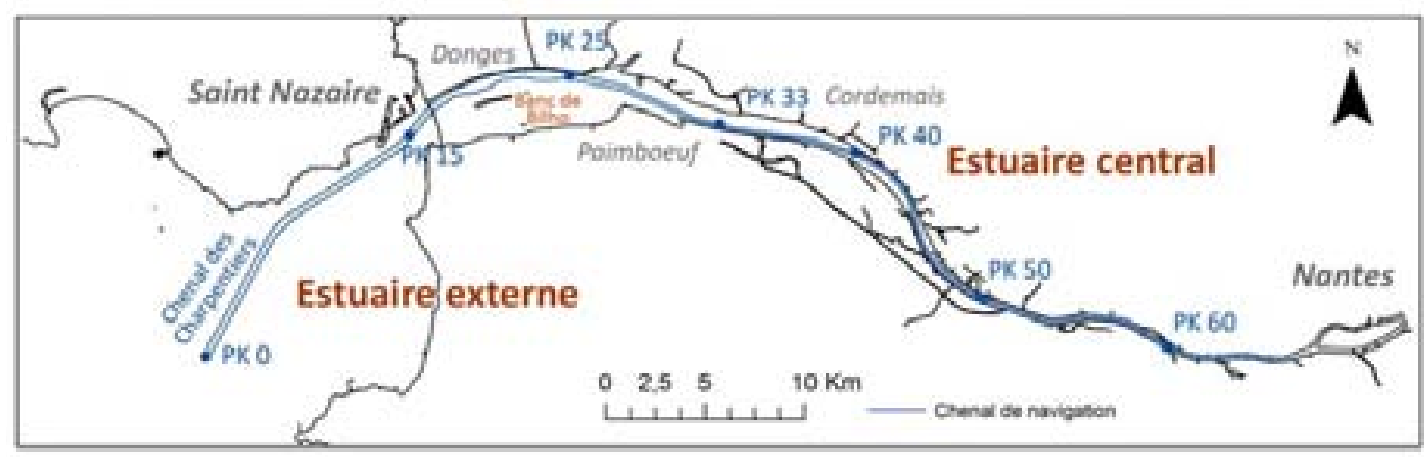

Figure 1. Carte toponymique de l'estuaire de Loire. 


\section{Matériel et méthodes}

\subsection{Base de données}

Le travail d'analyse s'est basé sur l'étude d'une collection de bandes d'enregistrement de sondages bathymétriques, mise à disposition par le Grand Port Maritime de Nantes Saint Nazaire (GPMNSN). Les mesures ont été acquises par les hydrographes du Port à l'aide d'un sondeur bifréquence $(33 / 210 \mathrm{kHz})$, au cours de transits et de profils réalisés entre 1997 et 2008. Les mesures sont enregistrées sur des bandes de papier thermique de plusieurs mètres de longueur. Ces bandes ont été prêtées par le Port pour cette étude.

Dans la partie amont, 127 sondages ont été analysés. Ils ont été levés entre 1998 et 2007, sur des transits situés dans l'axe et au centre du chenal de navigation, entre Nantes et Paimboeuf. La bande est positionnée dans l'espace grâce aux points kilométriques (référentiel du GPMNSN) qui sont notés sur la bande. En général, la mesure de cette zone est réalisée en une journée. La première analyse de ces données montre que $65 \%$ des profils mesurés présentent de la crème de vase. La figure 2 précise l'étendue de la crème de vase détectée sur les bandes, au cours du temps. La chronique du débit fluvial entre 1998 et 2007 est indiquée pour information.

Dans la partie située en aval de Paimboeuf, le Grand Port Maritime de Nantes SaintNazaire a réalisé des sondages bathymétriques constitués de séries de profils perpendiculaires au chenal. Les mesures de 2008 ont été prêtées pour l'étude. Les profils sont positionnées dans l'espace en fonction des points kilométriques du port et des limites du chenal de navigation qui sont notées sur la bande. L'estuaire aval étant décomposé en 7 sections, une journée de mesure permet en général de lever une section complète. Sur les 147 sondages étudiés dans la partie aval de l'estuaire, 90\% des enregistrements montrent la présence de crème de vase (figure 4).

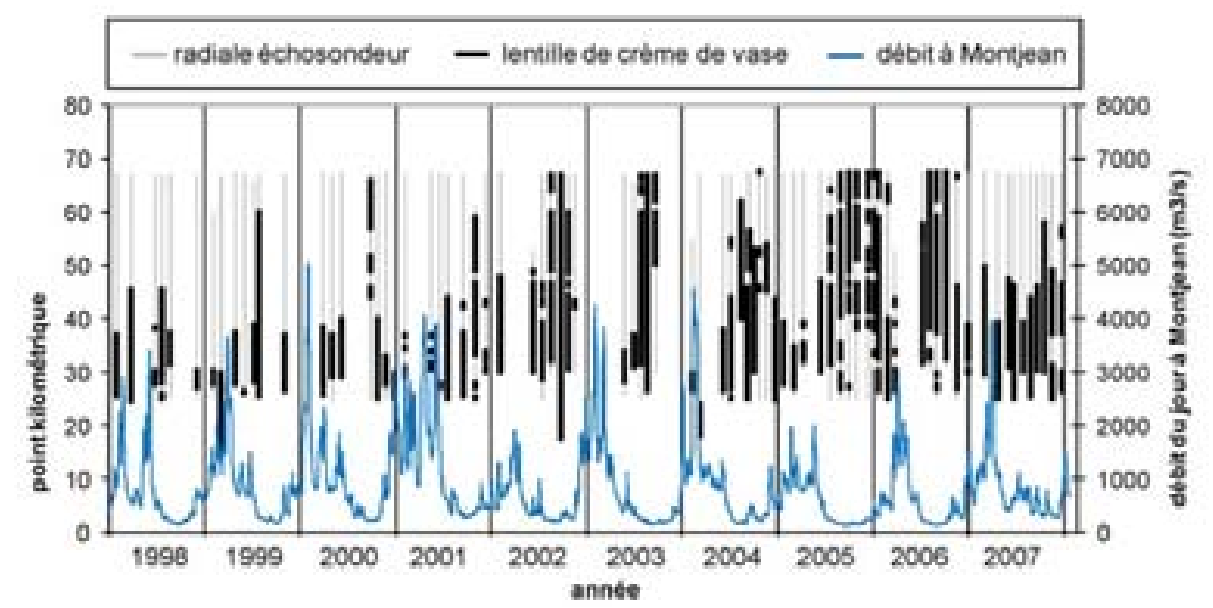

Figure 2. Extension des levés bathymétriques amont et des lentilles de crème de vase détectées en fonction du temps et du débit fluvial. 


\subsection{Analyse des faciès acoustiques}

L'examen poussé des bandes de sondages bathymétriques a également permis de repérer différentes interfaces de densité superposées, indiquant la stratification des dépôts en couches de concentration supposée homogène, séparées par des lutoclines (KIRBY, 1988). De plus, l'aspect du signal acoustique rétrodiffusé est différent selon les couches traversées, suggérant des faciès acoustiques corrélés à l'état de consolidation de la crème de vase. Ces faciès acoustiques ont donc été associés à des "types" de crème de vase, établis précédemment par la synthèse de nombreux profils verticaux de concentration obtenus par l'IFREMER au moyen de sondes OBS dans les estuaires de la Loire et de la Gironde (campagnes SEDIGIR, BASSOULLET \& LE HIR, 1997). La figure 3 montre un extrait d'enregistrement du sondeur bi-fréquence sur lequel on observe une superposition de faciès acoustiques associés à des couches de crème de vase de concentration croissante. Trois "types" de crème de vase ont ainsi été distingués : la crème de vase liquide (30 à $\left.100 \mathrm{~g} \mathrm{l}^{-1}\right)$, la crème de vase sensu stricto (100 à $\left.300 \mathrm{~g} \mathrm{l}^{-1}\right)$ et la crème de vase consolidée $\left(\mathrm{C}>300 \mathrm{~g}^{-1}\right)$.

Dans la partie centrale de l'estuaire, les faciès acoustiques de 30 sondages ont été analysés finement afin d'établir l'épaisseur, la stratification et la répartition des différents types de crème de vase pour une large gamme de conditions hydrologiques. Dans la partie externe, les différents faciès repérés ont été notés pour chaque section, comme le montre la figure 4. La chronique du débit fluvial et l'évolution du coefficient de marée au cours de l'année 2008 sont également indiquées. Puis, deux séries de mesures ont été analysées en détail pour établir la répartition et les épaisseurs des différents faciès de crème de vase au cours de conditions hydrologiques contrastées (étiage et crue).

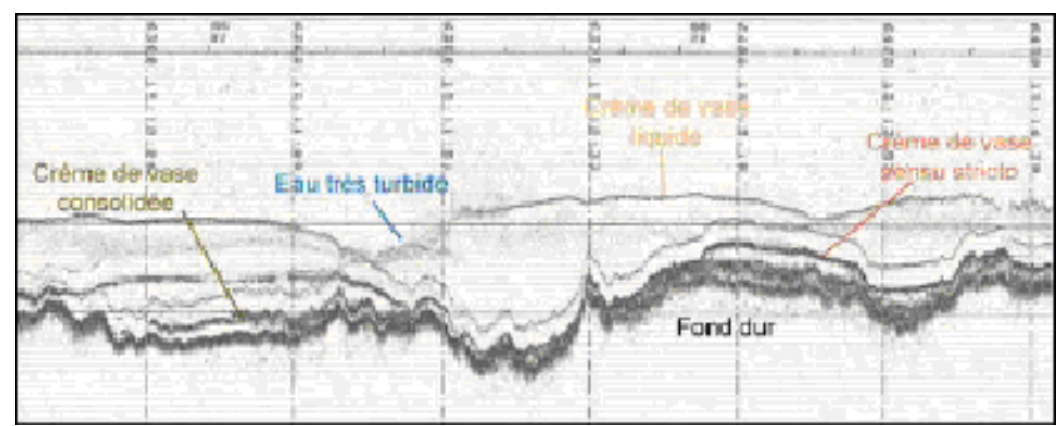

Figure 3. Les différents faciès acoustiques observés sur les sondages bathymétriques.

\section{Résultats}

\subsection{Partie centrale}

Dans la partie centrale de l'estuaire, le travail sur une base de donnée étendue a permis d'établir un "catalogue" de dépôt de crème de vase. L'étude des situations de dépôt doit 


\section{XI $I^{\text {èes }}$ Journées Nationales Génie Côtier - Génie Civil \\ Les Sables d'Olonne, 22-25 juin 2010}

permettre de mieux comprendre les conditions de formation et d'évolution des lentilles de crème de vase. Trois situations caractéristiques peuvent être distinguées.

a) Débit moyen et vive-eau

La situation du $1^{\text {er }}$ août 2007 (figure 5) est observée après que le débit fluvial se soit stabilisé depuis trois semaines autour de $700 \mathrm{~m}^{3} / \mathrm{s}$. La crème de vase s'est concentrée dans le secteur situé entre Cordemais et Donges. La crème de vase est très souvent présente dans cette zone située au niveau d'un seuil bathymétrique. Les courants de vive-eau favorisent le maintien de crème de vase liquide, formant ponctuellement des couches d'épaisseur supérieur à $2 \mathrm{~m}$.

b) Etiage et morte-eau

Le dépôt observé le 13 août 2004 correspond à une période d'étiage (figure 5). Le débit du fleuve est resté inférieur à $250 \mathrm{~m}^{3} / \mathrm{s}$ pendant près de deux mois. Le bouchon vaseux et la crème de vase associée sont remontés dans l'estuaire jusqu'à Nantes. Après une semaine de morte-eau, les dépôts ont commencé à se tasser, ce qui explique l'absence de crème de vase liquide. Entre Nantes et Le Pellerin, de grandes épaisseurs de crème de vase sont stockées dans une succession de fosses.

c) Débit soutenu

La situation du 2 février 1999 (figure 5) correspond à une mesure effectuée douze jours après le passage d'une crue à $2050 \mathrm{~m}^{3} / \mathrm{s}$, avec un débit qui reste soutenu à $1500 \mathrm{~m}^{3} / \mathrm{s}$. La crème de vase s'est déplacée dans un domaine de profondeur plus importante, vers la partie aval de l'estuaire. De la vase consolidée reste toutefois observée entre Donges et Paimboeuf, dans la principale zone de dépôt de l'estuaire amont.

\subsection{Partie externe}

La partie externe de l'estuaire de la Loire est un domaine soumis à une pression de dragage intense pour maintenir la profondeur du chenal et les souilles le long des quais. En 2008 le volume dragué dans ce secteur est estimé à $10.10^{6} \mathrm{~m}^{3}$. Le remaniement des sédiments par ces dragages pourrait faire apparaître des lentilles de crème de vase qui ne sont pas liées au bouchon vaseux Dans cette zone, la cartographie basée sur l'analyse de profils perpendiculaires au chenal permet de décrire précisément la morphologie des dépôts de crème de vase situés dans le chenal de navigation.

a) Débit soutenu

La situation observée entre le 4 et le 14 février 2008 (figure 6) correspond à des mesures réalisées plus de deux semaines après une crue à $2000 \mathrm{~m}^{3} / \mathrm{s}$, pendant une période de débit soutenu supérieur à $1200 \mathrm{~m}^{3} / \mathrm{s}$. Dans ces conditions, de très fortes épaisseurs de crème de vase sont observées devant Donges et devant Paimboeuf, comme pour la situation du 2 février 1999. Plus en aval, au-delà du pont de Saint Nazaire, le fond du chenal de navigation présente une épaisseur de crème de vase d'environ $1 \mathrm{~m}$, traduisant des dépôts liés aux phénomènes d'expulsion du bouchon vaseux dans la partie externe. 


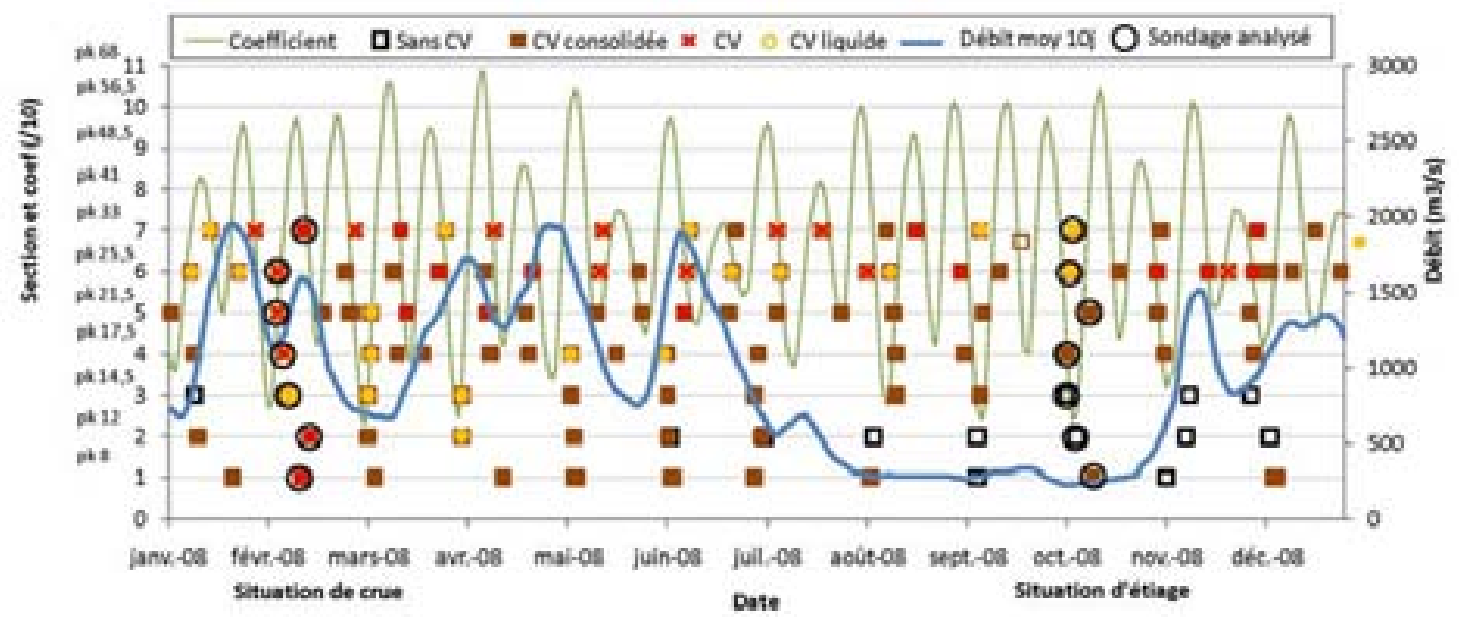

Figure 4. Faciès de crème de vase par section, dans la partie aval de l'estuaire, en fonction du temps, du débit fluvial et des coefficients de marée.

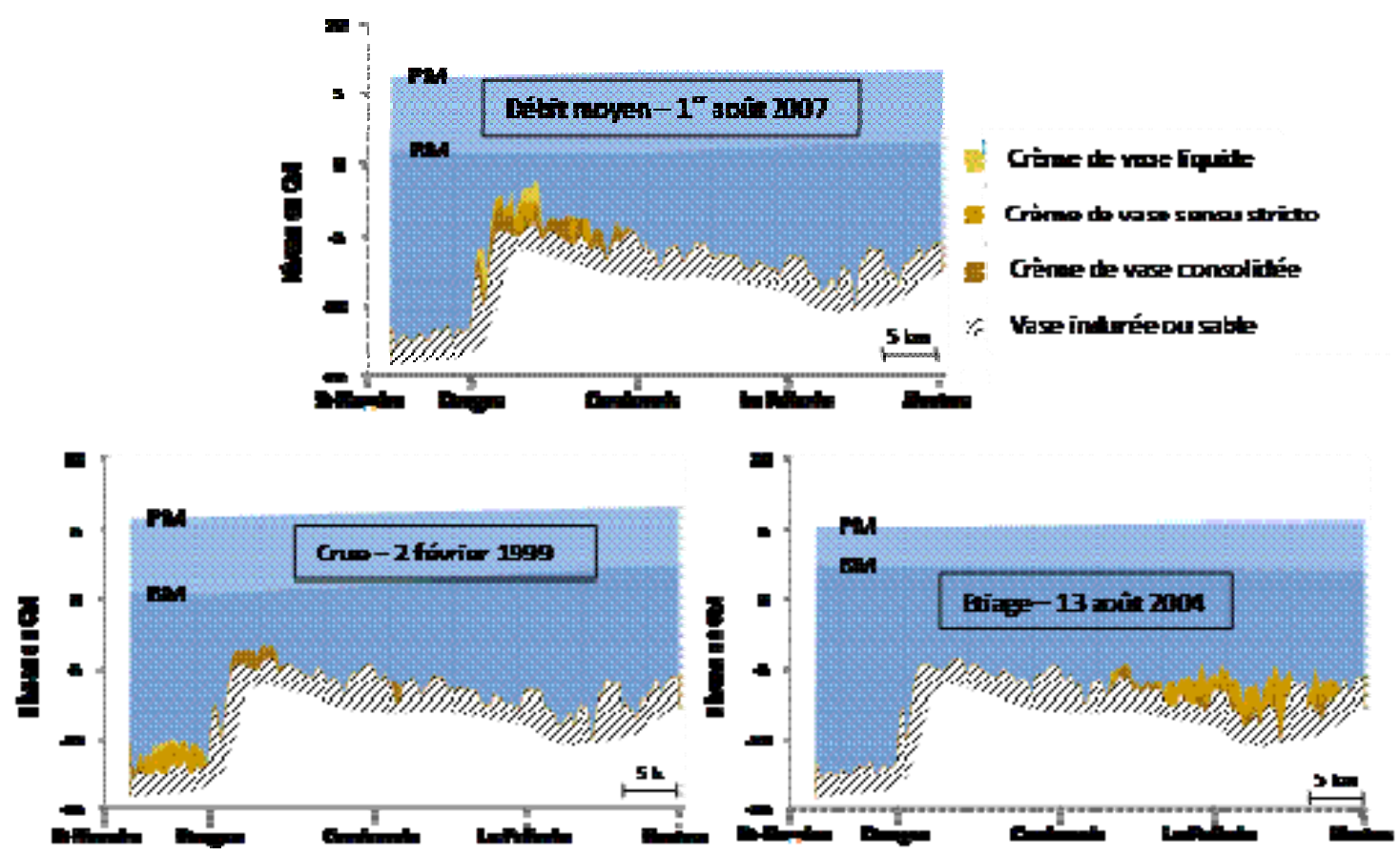

Figure 5. Dépôts de crème de vase dans la partie centrale de l'estuaire de la Loire, pour trois situations caractéristiques.

\section{b) Etiage}

La situation observée entre le 6 et le 14 octobre 2008 correspond à une longue période de faibles débits, inférieurs à $300 \mathrm{~m}^{3} / \mathrm{s}$. La figure 7 montre la présence de lentille de crème de vase consolidée d'épaisseur supérieur à $1 \mathrm{~m}$ entre Donges et Saint Nazaire. Des dépôts de crème de vase inférieurs à $1 \mathrm{~m}$ d'épaisseur se retrouvent également à la limite extérieure du chenal de navigation entretenu par dragage. La présence de ces 


\section{XI $I^{\text {èmes }}$ Journées Nationales Génie Côtier - Génie Civil}

Les Sables d'Olonne, 22-25 juin 2010

lentilles de crème de vase consolidée n'est pas liée au bouchon vaseux qui, en condition d'étiage, se situe bien en amont de la partie extérieure de l'estuaire de la Loire. Au niveau de la section 6 et en amont, elles pourraient être issues de la mise en œuvre, peu de temps avant les mesures, d'une technique de déconsolidation des sédiments du chenal par injection d'eau (Jetsed). Au niveau des sections 4 et 5 , la présence de crème de vase semble liée à l'entretien des souilles d'appontage par une drague refoulant les sédiments dans le chenal.

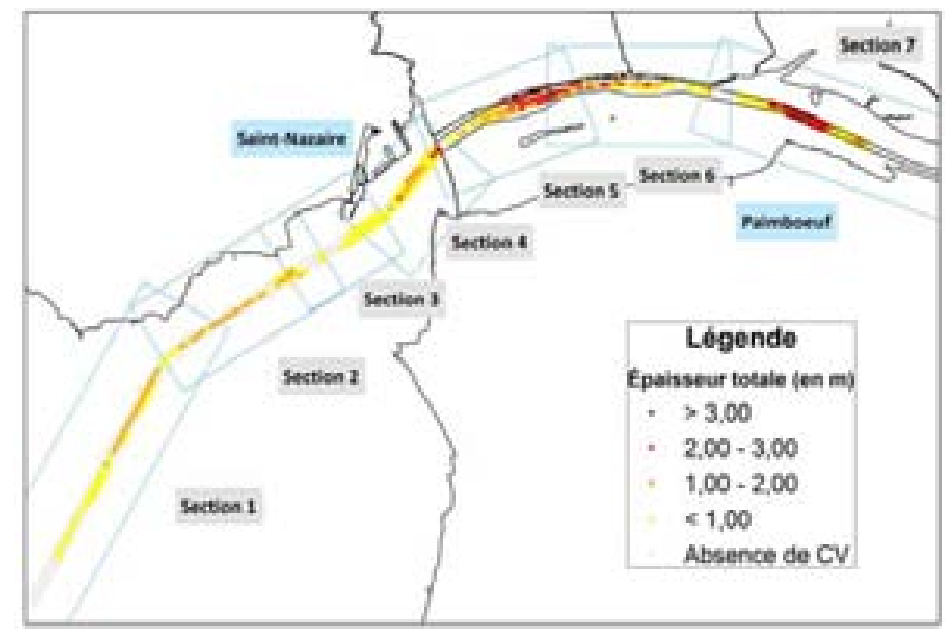

Figure 6. Epaisseur des dépôts de crème de vase dans la partie aval de l'estuaire de la Loire. Situation de crue entre le 4 et le 14 février 2008.

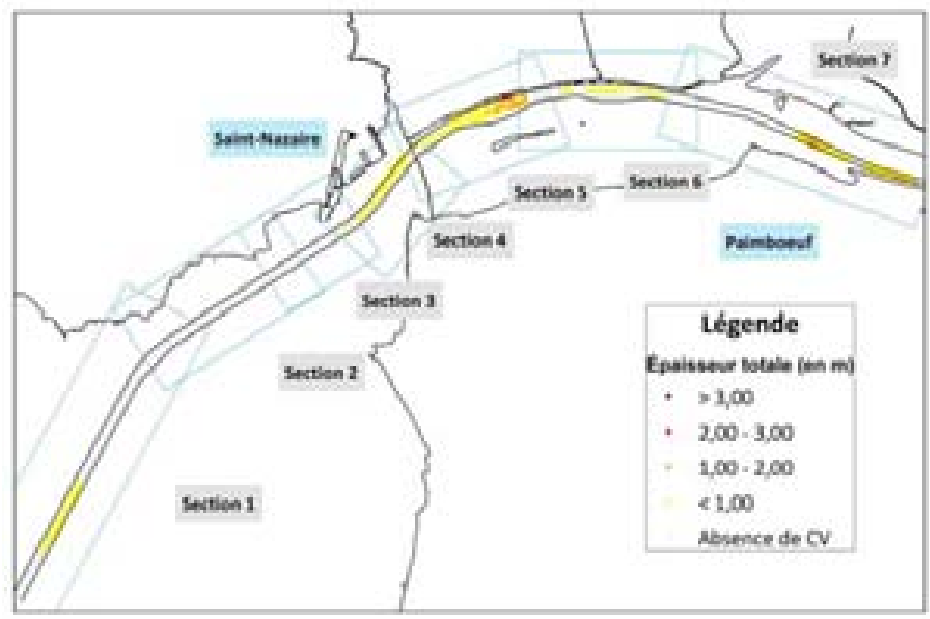

Figure 7. Epaisseur des dépôts de crème de vase dans la partie aval de l'estuaire de la Loire. Situation d'étiage entre le 6 et le 14 octobre 2008.

\section{Conclusions}

Cette étude a permis d'analyser une large base de données bathymétriques acquise entre 1998 et 2008 par le GPMNSN. Les premiers résultats mettent en évidence une relation 
forte entre les débits fluviaux et les caractéristiques des dépôts. En revanche, à notre échelle d'observation, l'étude des mesures de sondeur bifréquence ne montre pas d'influence prépondérante des coefficients de marée, contrairement aux travaux de GALLENNE (1974). Différentes situations ont été analysées finement. Le travail sur les signaux acoustiques enregistrés a permis de cartographier l'épaisseur de chaque faciès afin d'obtenir un catalogue de situations de dépôt. Néanmoins, les caractéristiques sédimentaires (concentration, tassement, etc.) des faciès acoustiques de crème de vase doivent encore être validées en calibrant le sondeur bifréquence avec les sédiments de la Loire. Les cartes établies montrent qu'au delà d'un lien fort entre la crème de vase et la position du bouchon vaseux, certaines zones situées devant Donges ou Paimboeuf semblent favoriser cette sédimentation. De même, le chenal de navigation situé en aval de Saint Nazaire apparait affecté tout au long de l'année par la présence de vase molle, y compris en période d'absence du bouchon vaseux dans l'estuaire aval. Les activités de dragage et de clapage peuvent être à l'origine de ces lentilles de crème de vase. Enfin, cette étude a permis de comprendre la nécessité de prendre en compte l'historique du dépôt et sa dynamique à plus court terme pour pouvoir mieux expliquer la structure et la forme des lentilles de crème de vase.

\section{Références bibliographiques}

BASSOULLET P., LE HIR P. (1997). Mesures hydrosédimentaires in-situ dans l'entité bouchon vaseux-crème de vase. Rapport Intermédiaire URM 13 Ifremer-U. Bordeaux 1CNRS «Evolution environnementale des systèmes Arcachon-Gironde », coordination P. Castaing et A. Romaña, pp 93-113.

GALLENNE B. (1974). Les accumulations turbides dans l'estuaire de la Loire - Etude de la "crème de vase". Thèse, Université de Nantes, 323 p.

KIRBY R. (1988) High concentration suspension (fluid mud) layers in estuaries. Physical Processes in Estuaries, L.J. Dronkers, W. Van Leussen (Eds.), SpringerVerlag, pp 463-487.

MAURICE L. (1994) Biodégradabilité de la matière organique dans le bouchon vaseux et le crème de vase de l'estuaire de la Loire. Oceanologica Acta 17 (5), pp 501-516.

MIGNIOT C. (1993). Bilan de l'hydrologie et de l'hydrosédimentaire de l'estuaire de la Loire au cours des deux dernières décennies. APEEL - PA Nantes - Saint Nazaire.

ROSS M.A., MEHTA A.J. (1989). On the mechanics of lutoclines and fluid mud. J. of Coastal Research, Sp issue 5, High Concentration Cohesive Sediment Transport, A.J. Mehta, E.J. Hayter (Eds.), pp 51-61.

SANCHEZ M., DELANOË Y. (2006). L'envasement dans différents ports de LoireAtlantique. IX ${ }^{\text {èmes }}$ Journées Nationales Génie Civil - Génie Côtier, Brest, pp 419-430. doi:10.5150/jngcgc.2006.040-S 